\title{
PREVALENCIA DE SINTOMATOLOGÍA OSTEOMUSCULAR EN MIEMBRO SUPERIOR Y FATIGA LABORAL EN UNA EMPRESA DE MANUFACTURA DE LA CIUDAD DE CALI
}

\author{
PREVALENCE OF MUSCULOSKELETAL SYMPTOMS IN THE UPPER LIMB AND WORK \\ FATIGUE IN A MANUFACTURING COMPANY IN THE CITY OF CALI
}

\author{
Jessica López Laverde ${ }^{1}$
}

\begin{abstract}
Resumen: La fatiga muscular es un fenómeno presente en la actividad laboral, lo mismo que la sintomatología dolorosa osteomuscular, generalmente, asociadas a actividades de fuerza, movimientos repetitivos y posturas prolongadas o forzadas. En una empresa manufacturera de la ciudad de Cali, los reportes por ausentismo laboral asociados a estas condiciones, fueron reiterativos en los últimos años. A partir del reconocimiento de estas condiciones, surge la necesidad de identificar el estado ergonómico, con el fin de poder establecer las respectivas recomendaciones. Se aplicó el cuestionario Nórdico, que arrojó una prevalencia para sintomatología dolorosa osteomuscular en cincuenta y cinco operarias, de las cuales veintiuna presentaron dolor en los últimos siete días antes de aplicar el instrumento, y veinticuatro operarias, a quienes el dolor limitó en las actividades diarias, con incapacidad médica en nueve de estas. Además, se utilizó el cuestionario de síntomas subjetivos de Yoshitake validado para fatiga laboral, arrojando que la fatiga física y el nivel preponderante de fatiga fue severa. Así mismo, se halló una asociación significativa entre sintomatología dolorosa osteomuscular y fatiga laboral, respecto de sintomatología dolorosa osteomuscular en hombro y nivel de fatiga leve al inicio de la jornada laboral. A la par se ejecutó una lista de chequeo ergonómica, con la que se evaluó las características del ambiente físico, las máquinas y herramientas. Después del análisis de los resultados, se realizaron recomendaciones para mejorar las condiciones de trabajo.
\end{abstract}

Palabras clave: Sintomatología dolorosa osteomuscular, fatiga laboral, cuestionario de Yoshitake, cuestionario Nórdico.

\begin{abstract}
Muscular fatigue is a phenomenon present in the work activity, the same as osteomuscular pain symptomatology. Generally, associated with activities of force, repetitive movements and prolonged or forced postures. In a manufacturing company in the city of Cali, reports of absenteeism associated with these conditions were reiterative in recent years. From the recognition of these conditions, the need arises to identify the ergonomic state, in order to be able to establish the respective recommendations. For which, the Nordic questionnaire was applied, which showed a prevalence for painful osteomuscular symptoms in fifty five operators, of which twenty one presented pain in the last seven days prior the application of the instrument and twenty four operators whose pain limited their daily activities, with medical incapacity in nine of these. In addition, the Yoshitake's subjective symptom questionnaire validated for work fatigue was used, showing that the physical fatigue and the prevailing level of fatigue was severe. Likewise, a significant association was found
\end{abstract}

IInstitución Universitaria Escuela Nacional del Deporte. Cali, Colombia. Correo electrónico: jessica.lopez@endeporte.edu.co. Orcid: https://orcid.org/oooo-0003-0412-0803 
between painful osteomuscular symptoms and work fatigue, with respect to osteomuscular pain symptomatology in the shoulder and mild fatigue level at the beginning of the work day. At the same time, an ergonomic checklist was executed to evaluate the characteristics of physical environment, machines and tools. After the analysis of the results, recommendations were made to improve working conditions.

Keywords: Painful musculoskeletal symptoms, work fatigue, Yoshitake's questionnaire, Nordic questionnaire.

Recepción: 02.11.2020 / Revisión: 04.11.2020 / Aceptación: 02.12.2020

\section{Introducción}

Una primera condición para la aparición de desórdenes musculoesqueléticos (DME), es la presencia de sintomatología dolorosa osteomuscular y fatiga muscular, siendo estas las causas más frecuentes de patologías de origen laboral. Por tanto, se requieren estudios que permitan de manera adecuada evidenciar estos síntomas.

En la empresa manufacturera estudiada, se empezó a recibir reportes verbales de las operarias, como cansancio, pesadez, monotonía, falta de atención, y dolor osteomuscular, lo que llevó a considerar la importancia de evidenciar estas condiciones.

Se debe considerar que la fatiga, se encuentra asociada al aporte de energía muscular y la eliminación de toxinas del cuerpo; su principal causa es la actividad física prolongada y exigente. Esta se caracteriza por la presencia de dolor agudo en los músculos. En la aparición de la fatiga laboral, se suman varias causas; entre estas se encuentran los factores relativos a las condiciones físicas (ruido, temperatura, iluminación), los factores psicosociales (relaciones jerárquicas, sistema de comunicación, etc.), y los factores de origen extralaboral (Cubillos \& López, 2009).

Para este estudio, se utilizó el cuestionario de síntomas o test de Yoshitake en cuanto a la variable fatiga subjetiva. Siendo un cuestionario de síntomas subjetivos de fatiga, que se aplica antes y después de la jornada laboral. El comité para la investigación de la fatiga industrial, de la Asociación de Salud Industrial de Japón en 1954, desarrolló un instrumento que consta de 30 reactivos que exploran la presencia de síntomas que originalmente fueron clasificados en tres grupos: físicos, mentales y neurosensoriales. Saito, Kogi y Kashifawi, en 1970 sometieron a validez factorial el instrumento, obteniendo tres factores: somnolencia y pesadez, proyección de malestar físico y dificultad para concentrarse. Yoshitake en 1978 relacionó el primer factor con trabajo indiferenciado, el segundo con trabajo físico y el tercero con trabajo mental; adicionalmente propuso la calificación de la prueba a través del porcentaje de respuestas afirmativas (Araujo \& Alhejandra, 2014).

También se aplicó el cuestionario Nórdico estandarizado, elaborado y propuesto a la comunidad científica internacional en el año 1987 tras su validación en la población de referencia de los autores (población escandinava). Se trata de una herramienta cuyo uso se ha extendido ampliamente en los últimos años en todos los países desarrollados ya que ha demostrado poseer una extraordinaria utilidad a la hora de estudiar sintomatología 
musculoesquelética en población trabajadora y en diferentes localizaciones anatómicas (Rengifo-Villavicencio, 2020).

Además, se aplicó una lista de chequeo, elaborada en base a recopilación de información inherente al área evaluada, por ser una herramienta tangible que funcionó como una lista de factores claves para estudiar, observar y cualificar el estado ergonómico del puesto de trabajo, postura, condiciones locativas, eléctricas, ambiente en general, entre otros. Todo lo anterior, con el fin de establecer los criterios ergonómicos para la prevalencia de sintomatología osteomuscular en miembro superior y fatiga laboral en el área de punto y plano en una empresa de manufactura de la ciudad de Cali.

\section{Materiales y métodos}

Se realizó un estudio descriptivo, transversal no experimental. Para el desarrollo de este trabajo se efectuaron visitas de campo a la empresa manufacturera. Se tomó un registro fotográfico de las tareas realizadas, con el fin de establecer los puestos de trabajo, posturas, entorno laboral, entre otros. Simultáneamente, se aplicaron los instrumentos a cincuenta y cinco operarias.

El cuestionario de sintomatología dolorosa osteomuscular (SDO) fue aplicado de manera auto administrada, aleatoria y voluntaria a aquellas personas que decidieron participar. Previo a la aplicación del cuestionario, se desarrolló una socialización de 5 minutos sobre el mismo. No obstante, hubo un sesgo de información, que aparece en los resultados como no "aplica", debido a la falta de completitud de algunos cuestionarios.

Se aplicó, también el cuestionario de Yoshitake, que determina la presencia o ausencia de la fatiga por medio de 30 preguntas, divididas en tres grupos de preguntas, el primero consta de 10 preguntas relacionadas con síntomas de somnolencia y monotonía, el segundo de 10 preguntas relacionadas con la dificultad de concentración y el tercero con 10 preguntas respecto de síntomas corporales o proyección de daño físico. De esta manera, se considera un estado de fatiga cuando se alcanzan siete síntomas positivos (23,33\%) en mujeres y seis síntomas en hombres (20\%). Este instrumento diferencia los siguientes tipos de fatiga laboral: tipo I, corresponde a fatiga general o mixta (ítem 1 al 10= P1), tipo II corresponde a fatiga intelectual (ítem del 11 al $20=\mathrm{P} 2$ ) y tipo III a fatiga física (ítem del 21 al $30=\mathrm{P}_{3}$ ). Se determinará fatiga tipo 1, sí P1 > P2 y P2 > P3; fatiga tipo 2, si $\mathrm{P}_{2} \geq \mathrm{P} 1 \geq \mathrm{P}_{3}$ y fatiga tipo 3, si $\mathrm{P}_{3} \geq \mathrm{P} 1 \geq \mathrm{P} 2$. Además, el cuestionario determina el nivel de fatiga a través del puntaje que se otorga a las respuestas afirmativas; 6 respuestas positivas corresponden a fatiga leve, de 7 a 12 fatiga moderada y de 13 a 30 fatiga severa. (Gómez-Cajas, 2014). Este cuestionario tuvo por objetivo establecer la fatiga laboral, este se utilizó con la misma población a la cual se le aplicó el Nórdico. El cuestionario de Yoshitake se efectuó en dos momentos durante la jornada laboral, previo a la socialización del instrumento. A diferencia del Nórdico, el acompañamiento fue personalizado, se contó con el apoyo de nueve personas entrenadas en la aplicación del instrumento, incluida la investigadora. Como se describió anteriormente, consta de 30 preguntas, las cuales exploran síntomas generales de fatiga, fatiga mental y física, las cuales están caracterizadas de la siguiente forma (Escorza, 2010): 
- Síntomas generales de fatiga: pesadez en la cabeza, cansancio en el cuerpo, torpeza, somnolencia y deseos de acostarse.

- Fatiga mental: caracterizada por la dificultad para pensar, nerviosismo, problemas en la atención y deseos de acostarse.

- Fatiga física: incluye dolor de cabeza, dolores en el cuerpo y cansancio físico general.

De igual manera, para el abordaje de la relación entre las variables de sintomatología dolorosa osteomuscular y fatiga laboral, se contó con el programa SPSS Statistics 21 para Windows, que permite explorar visualmente la relación entre dos variables cuantitativas a través de gráficos (Aguayo-Canela, 2007).

Se elaboró una lista de chequeo, con base a recopilación de algunas de estas, como una herramienta de seguimiento en forma de cuestionario y con un listado de preguntas, para verificar el grado de cumplimiento de las condiciones físicas y ergonómicas del área evaluada. Las listas de chequeo comenzaron a utilizarse en el ámbito parlamentario, en Alemania a mediados de la década del setenta y rápidamente su uso se extendió a otros países como Suiza, Noruega, EUUU y Francia (Bichachi, 2010).

Finalmente, con el análisis de los resultados de los instrumentos y la lista de chequeo, se realizan las respectivas recomendaciones.

\section{Resultados y discusión}

\section{Prevalencia de sintomatología dolorosa osteomuscular en miembro superior}

Se aplicó el cuestionario Nórdico modificado, el cual permitió estimar el nivel de sintomatología dolorosa para el segmento de miembro superior. Cabe resaltar que, a pesar de ser el cuestionario anónimo, se hallaron algunos cuestionarios sin completar preguntas, por lo que algunas tablas en su presentación, aparecen con una columna, de un "no aplica". A continuación, las tablas y gráficas.

Tabla 1. Sintomatología dolorosa en miembro superior.

\begin{tabular}{|l|c|}
\hline Sintomatología dolorosa en miembro superior & Sí \\
\hline Hombro & 19 \\
\hline Codo & 6 \\
\hline Mano/muñeca & 30 \\
\hline Total & 55 \\
\hline
\end{tabular}


Tabla 2. Dolor en los últimos 7 días por segmento del miembro superior.

\begin{tabular}{|l|c|c|c|c|}
\hline Segmento & No & Sí & No aplica & Total \\
\hline Hombro & 7 & 7 & 5 & $\mathbf{1 9}$ \\
\hline Codo & 4 & 2 & 0 & $\mathbf{6}$ \\
\hline Mano/muñeca & 15 & 12 & 3 & $\mathbf{3 0}$ \\
\hline
\end{tabular}

Se encuentra que la SDO es más prevalente en el segmento de dedos y muñeca, lo que pudiese ser debido a la actividad laboral que está relacionada con motricidad fina.

Tabla 3. Limitación para actividades de la vida diaria por segmento del miembro superior.

\begin{tabular}{|l|c|c|c|c|}
\hline Segmento & No & Sí & No aplica & Total \\
\hline Hombro & 8 & 9 & 2 & $\mathbf{1 9}$ \\
\hline Codo & 6 & 0 & 0 & $\mathbf{6}$ \\
\hline Mano/muñeca & 14 & 10 & 6 & $\mathbf{3 0}$ \\
\hline
\end{tabular}

Igualmente, el segmento que más ha generado limitación en las actividades cotidianas es la muñeca y la mano.

Tabla 4. Incapacidad médica por dolor por segmento del miembro superior.

\begin{tabular}{|l|c|c|c|c|}
\hline Segmento & No & Sí & No aplica & Total \\
\hline Hombro & 15 & 4 & 0 & $\mathbf{1 9}$ \\
\hline Codo & 6 & 0 & 0 & $\mathbf{6}$ \\
\hline Mano/muñeca & 23 & 5 & 2 & $\mathbf{3 0}$ \\
\hline
\end{tabular}

Se observa que la incapacidad mayormente está relacionada con el segmento de la muñeca y mano.

De las cincuenta y cinco operarias a las cuales se les aplicó el cuestionario, sólo veintiuna consideraron presencia de dolor, veinticuatro respondieron que les limita las actividades de la vida diaria, y solo nueve han presentado incapacidad laboral. Este resultado pudiese estar asociado a que, en el momento del estudio, se hallaban con alta producción, lo que para algunas genera la concesión de horas extras, además de las bonificaciones. Por lo demás, se encontró que el segmento más comprometido en cuanto a dolor es muñeca, esto probablemente asociado a las tareas repetitivas, y conjuntamente la altura de los planos de trabajo respecto de la antropometría de las operarias no permite una adecuada asociación.

En la observación, se encontró desviación de muñeca específicamente en la costura de algunas prendas, esto sin contar con los horarios que, para la época de abril, mayo y junio, 
meses en los cuales se realizó el estudio, suelen extenderse por acercarse la temporada de madre y verano; que pueden ir entre 9 y 10 horas diarias de lunes a sábado y medio tiempo el día domingo.

\section{Presencia de fatiga laboral}

Se aplicó el cuestionario Yoshitake en dos momentos, el cual arrojó los siguientes resultados.

Tabla 5. Tipo de fatiga.

\begin{tabular}{|l|c|c|c|c|}
\hline Tipo de fatiga & Normal & Fatiga mental & Fatiga física & Fatiga mixta \\
\hline Inicio de la jornada laboral & $\mathrm{O}$ & $\mathrm{O}$ & 49 & $\mathbf{6}$ \\
\hline Final de la jornada laboral & $\mathrm{O}$ & 3 & $\mathbf{2 5}$ & $\mathbf{2 7}$ \\
\hline Promedio & $\mathrm{o}$ & $\mathbf{1 . 5}$ & 37 & $\mathbf{1 6 , 5}$ \\
\hline
\end{tabular}

Los resultados respecto del cuestionario de síntomas subjetivos de Yoshitake, referente al tipo de fatiga al inicio de la jornada, arrojó que de las cincuenta y cinco operarias, el 100\% se encuentra con fatiga de tipo física y mixta, con predominio de una elevada fatiga física en un 89\%. Lo mismo sucedió al final de la jornada, pero con mayor resultado en ambos tipos de fatiga.

Se sacó un promedio en las dos jornadas respecto de los tipos de fatiga, que permitiera apreciar al inicio y al final de la jornada cómo se observan los tipos de fatiga. La fatiga física fue la de mayor predominio, posiblemente asociada a las amplias horas de trabajo, la falta de descansos, la postura en la máquina, y sumado a esto, la sobrecarga laboral por la temporada, que puede llevar a afectar la capacidad física de las operarias. De acuerdo a la Universidad Complutense de Madrid, la fatiga constituye un fenómeno complejo que se caracteriza porque el trabajador baja el ritmo de actividad, nota cansancio, ejecuta movimientos más torpes e inseguros, percibe una sensación de malestar e insatisfacción, y disminuye el rendimiento en cantidad y calidad.

Tabla 6. Nivel de fatiga.

\begin{tabular}{|l|c|c|c|c|}
\hline Nivel de fatiga & Normal & Fatiga leve & $\begin{array}{c}\text { Fatiga } \\
\text { moderada }\end{array}$ & Fatiga severa \\
\hline Inicio de la jornada laboral & 0 & 10 & 13 & $\mathbf{3 2}$ \\
\hline Final de la jornada laboral & 0 & 1 & 8 & $\mathbf{4 6}$ \\
\hline Promedio & 0 & 5,5 & 10,5 & $\mathbf{3 9}$ \\
\hline
\end{tabular}

Se puede observar en los resultados que el nivel de fatiga predominante, es aquella que se encuentra en una etapa "severa", al inicio y final de la jornada, en treinta y dos y cuarenta y seis operarias respectivamente, representando un promedio durante las jornadas de 39 
trabajadoras, lo que equivale al $71 \%$.

\section{Condiciones físicas y ergonómicas del área manufacturera}

A través de la lista de chequeo se encontró:

- Falta de orden y de limpieza en el lugar de trabajo por la distribución de telas en el poco espacio del pasillo.

- El espacio del área es insuficiente para las máquinas, mobiliarios y otros instrumentos de trabajo.

- La distancia libre mínima entre trabajadores no se cumple, el borde frontal de la mesa y el obstáculo más cercano detrás de cada trabajador es de $100 \mathrm{~cm}$.

- Falta demarcación en zonas, como los pasillos y sitios restringidos, además de la señalización que está pendiente en algunos puntos.

- Se entregan los elementos de protección, como tapa oídos. El problema es que no todas las operarias lo utilizan y no existe control sobre esto. El ruido en la planta, se encuentra por encima de los $70 \mathrm{dBA}$.

- El soplete de aire para las telas, genera vibración alrededor de las máquinas.

- Solo se encuentra una salida de emergencia, que es obstaculizada cuando aumenta la producción, pues las prendas y telas son colocadas en los pasillos.

- En su gran mayoría, las operarias cuentan con una silla de cuatro patas, de material no transpirable, las pocas sillas ergonómicas, tienen dañado el espaldar, además de la perilla que gradúa la inclinación.

- El plano de trabajo al no ser graduable, es alto para la mayoría de las operarias, lo que lleva a una posición en el miembro superior por fuera de ángulos de confort con flexión sostenida de hombro y desviaciones de muñeca.

- La postura prolongada sedente y el movimiento repetitivo son las posturas comunes.

- Son pocos los tiempos de recuperación y descanso.

- No existen suficientes bebederos, solo se cuenta con una fuente de agua.

\section{Relación entre la variable de sintomatología dolorosa osteomuscular y fatiga laboral}

A continuación se presentarán una serie de tablas de contingencia, las cuales pretenden mostrar la asociación entre las variables de sintomatología dolorosa osteomuscular y el tipo y nivel de fatiga al inicio y al final de la jornada laboral. Se muestran las tablas que permitieron revelar alguna evidencia significativa. Para esto se utilizó el SPSS Statistics 21. 
Tabla 7. Relación dolor hombro versus nivel de fatiga al inicio jornada laboral.

\begin{tabular}{|c|c|c|c|c|c|c|}
\hline & \multicolumn{2}{|c|}{ Dolor hombro } & \multirow{2}{*}{ Total } & \multirow{2}{*}{ Chi 2} \\
\hline & & & No & Sí & & \\
\hline \multirow{6}{*}{$\begin{array}{lr}\text { Nivel } & \text { de } \\
\text { fatiga } & \text { al } \\
\text { inicio } & \\
\text { jornada } & \end{array}$} & \multirow{2}{*}{ Leve } & Frecuencia & 7 & 3 & 10 & \multirow{8}{*}{$\begin{array}{c}1.662 \\
(0,445)\end{array}$} \\
\hline & & Porcentaje & $19,4 \%$ & $15,8 \%$ & $18,2 \%$ & \\
\hline & \multirow{2}{*}{ Moderada } & Frecuencia & 6 & 6 & 12 & \\
\hline & & Porcentaje & $16,7 \%$ & $31,6 \%$ & $21,8 \%$ & \\
\hline & \multirow{2}{*}{ Severa } & Frecuencia & 23 & 10 & 33 & \\
\hline & & Porcentaje & $63,9 \%$ & $52,6 \%$ & $60,0 \%$ & \\
\hline \multirow{2}{*}{\multicolumn{2}{|c|}{ Total }} & Frecuencia & 36 & 19 & 55 & \\
\hline & & Porcentaje & $100,0 \%$ & $100,0 \%$ & $100,0 \%$ & \\
\hline
\end{tabular}

Tabla 8. Relación dolor codo versus nivel de fatiga al inicio jornada laboral.

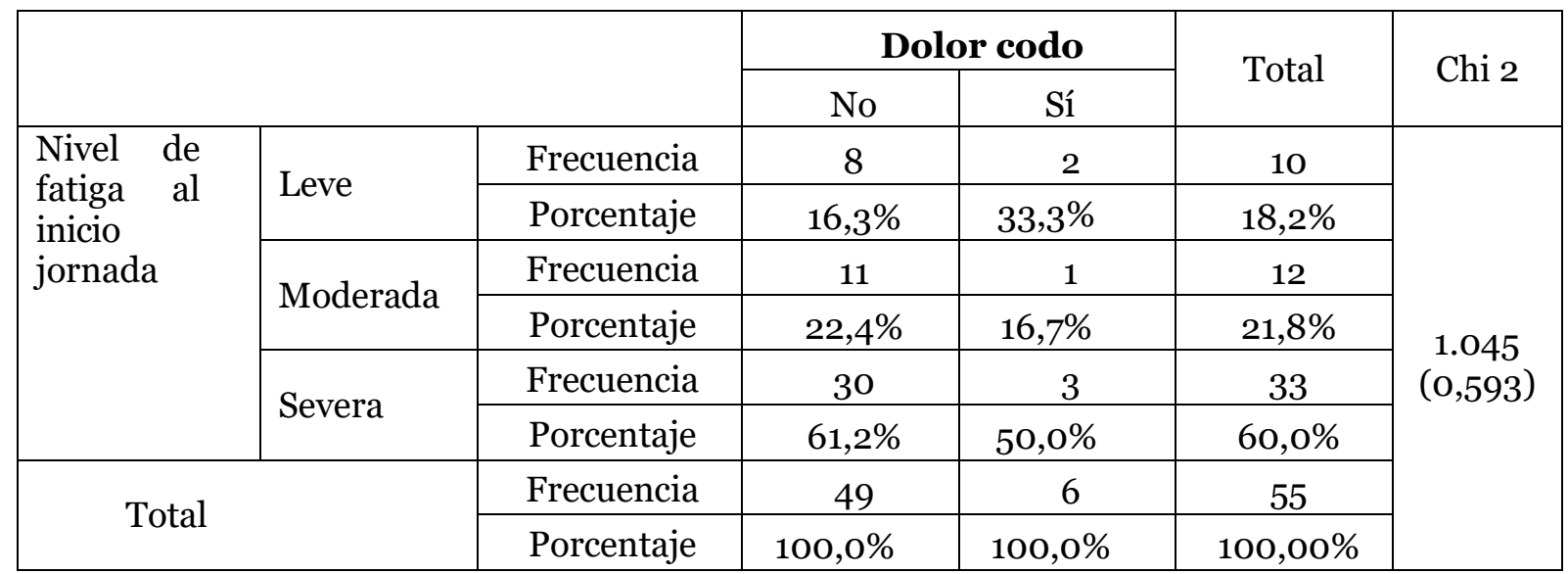

Tabla 9. Relación dolor muñeca versus nivel de fatiga al inicio jornada laboral.

\begin{tabular}{|c|c|c|c|c|c|c|}
\hline & \multicolumn{2}{|c|}{ Dolor muñeca } & \multirow{2}{*}{ Total } & \multirow{2}{*}{ Chi 2} \\
\hline & & & No & Sí & & \\
\hline \multirow{6}{*}{$\begin{array}{l}\text { Nivel de } \\
\text { fatiga al } \\
\text { inicio } \\
\text { jornada }\end{array}$} & \multirow{2}{*}{ Leve } & Frecuencia & 4 & 6 & 10 & \multirow{8}{*}{$\begin{array}{c}2.787 \\
(0,248)\end{array}$} \\
\hline & & Porcentaje & $16,0 \%$ & $20,0 \%$ & $18,2 \%$ & \\
\hline & \multirow{2}{*}{ Moderada } & Frecuencia & 8 & 4 & 12 & \\
\hline & & Porcentaje & $32,0 \%$ & $13,3 \%$ & $21,8 \%$ & \\
\hline & \multirow{2}{*}{ Severa } & Frecuencia & 13 & 20 & 33 & \\
\hline & & Porcentaje & $52,0 \%$ & $66,7 \%$ & $60,0 \%$ & \\
\hline \multirow{2}{*}{\multicolumn{2}{|c|}{ Total }} & Frecuencia & 25 & 30 & 55 & \\
\hline & & Porcentaje & $100,0 \%$ & $100,0 \%$ & $100,0 \%$ & \\
\hline
\end{tabular}

$\mathrm{Al}$ correlacionar la variable de dolor en hombro, codo y muñeca, se pudo evidenciar que al inicio de la jornada laboral, el nivel de fatiga que predomina en los tres segmentos, es el severo; esto probablemente asociado a las posturas mantenidas y repetitividad de la tarea, y a 
las largas jornadas del día anterior. No se halló asociación estadística entre estas.

Tabla 1o. Incapacidad hombro versus nivel de fatiga al inicio jornada laboral.

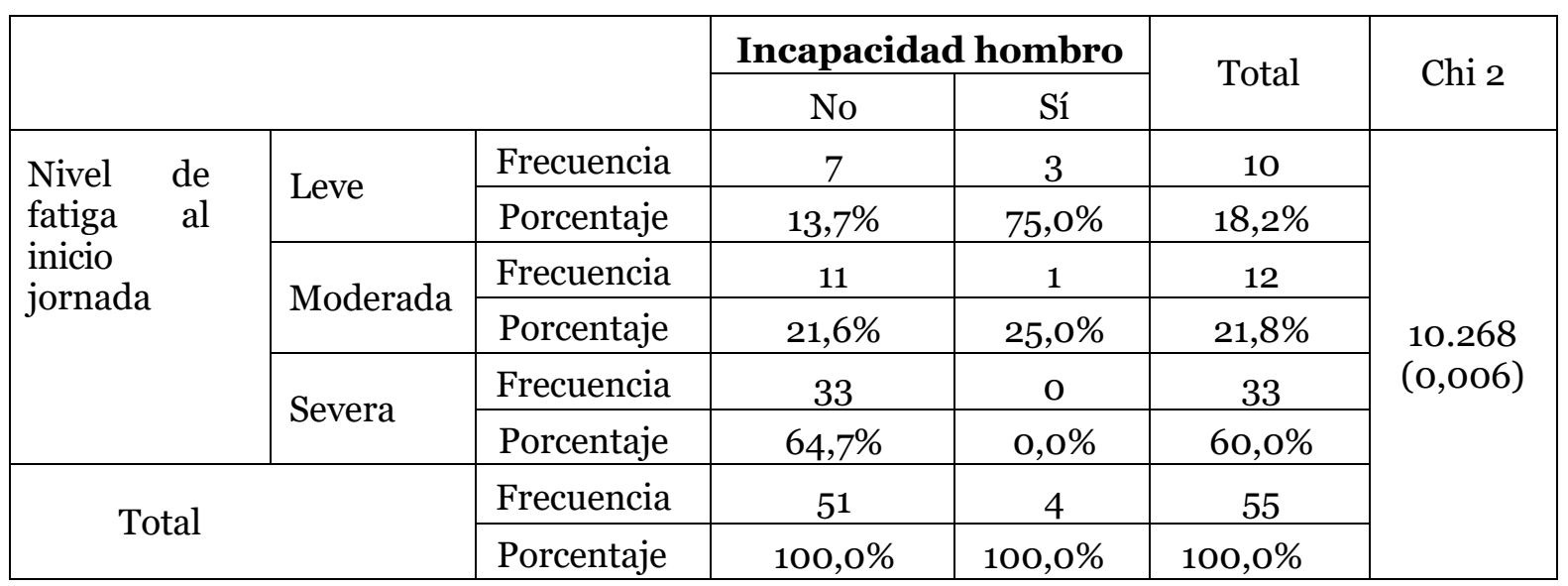

Se pudo evidenciar que, entre las variables incapacidad de hombro y nivel de fatiga leve, existe una asociación con significancia respecto de todas las tablas de contingencia del estudio. Probablemente esto esté relacionado con la incapacidad en hombro, el cual es un factor protector, que se presenta al inicio de la jornada laboral, como una fatiga leve, considerando que, en el segmento de muñeca, solo una operaria presenta fatiga leve.

Tabla 11. Incapacidad muñeca versus nivel de fatiga al inicio jornada laboral.

\begin{tabular}{|c|c|c|c|c|c|c|}
\hline & \multicolumn{2}{|c|}{ Incapacidad muñeca } & \multirow{2}{*}{ Total } & \multirow{2}{*}{ Chi 2} \\
\hline & & & No & Sí & & \\
\hline \multirow{6}{*}{$\begin{array}{lr}\text { Nivel } & \text { de } \\
\text { fatiga } & \text { al } \\
\text { inicio } & \\
\text { jornada } & \end{array}$} & \multirow{2}{*}{ Leve } & Frecuencia & 9 & 1 & 10 & \multirow{8}{*}{$\begin{array}{c}5.152 \\
(0,076)\end{array}$} \\
\hline & & Porcentaje & $18,0 \%$ & $20,0 \%$ & $18,2 \%$ & \\
\hline & \multirow{2}{*}{ Moderada } & Frecuencia & 9 & 3 & 12 & \\
\hline & & Porcentaje & $18,0 \%$ & $60,0 \%$ & $21,8 \%$ & \\
\hline & \multirow{2}{*}{ Severa } & Frecuencia & 32 & 1 & 33 & \\
\hline & & Porcentaje & $64,0 \%$ & $20,0 \%$ & $60,0 \%$ & \\
\hline \multirow{2}{*}{\multicolumn{2}{|c|}{ Total }} & Frecuencia & 50 & 5 & 55 & \\
\hline & & Porcentaje & $100,0 \%$ & $100,0 \%$ & $100,0 \%$ & \\
\hline
\end{tabular}

Se halló respecto de la incapacidad, que al inicio de la jornada el nivel de fatiga estaba entre leve y moderada, y solo una operaria presentó un nivel de fatiga severa para el segmento de la muñeca. 
Tabla 12. Relación dolor hombro versus tipo de fatiga inicio de la jornada laboral.

\begin{tabular}{|c|c|c|c|c|c|c|}
\hline & \multicolumn{2}{|c|}{ Dolor hombro } & \multirow{2}{*}{ Total } & \multirow{2}{*}{ Chi 2} \\
\hline & & & No & Sí & & \\
\hline \multirow{4}{*}{$\begin{array}{l}\text { Inicio de } \\
\text { jornada } \\
\text { tipo de } \\
\text { fatiga }\end{array}$} & \multirow{2}{*}{ Física } & Frecuencia & 32 & 17 & 49 & \multirow{6}{*}{$\begin{array}{c}0.004 \\
(0,947)\end{array}$} \\
\hline & & Porcentaje & $88,9 \%$ & $89,5 \%$ & $89,1 \%$ & \\
\hline & \multirow{2}{*}{ Mixta } & Frecuencia & 4 & 2 & 6 & \\
\hline & & Porcentaje & $11,1 \%$ & $10,5 \%$ & $10,9 \%$ & \\
\hline \multirow{2}{*}{\multicolumn{2}{|c|}{ Total }} & Frecuencia & 36 & 19 & 55 & \\
\hline & & Porcentaje & $100,0 \%$ & $100,0 \%$ & $100,0 \%$ & \\
\hline
\end{tabular}

Tabla 13. Relación dolor muñeca versus tipo de fatiga inicio de la jornada laboral.

\begin{tabular}{|c|c|c|c|c|c|c|}
\hline & \multicolumn{2}{|c|}{ Dolor muñeca } & \multirow{2}{*}{ Total } & \multirow{2}{*}{ Chi 2} \\
\hline & & & No & Sí & & \\
\hline \multirow{4}{*}{$\begin{array}{l}\text { Inicio de } \\
\text { jornada } \\
\text { tipo de } \\
\text { fatiga }\end{array}$} & \multirow{2}{*}{ Física } & Frecuencia & 24 & 25 & 49 & \multirow{6}{*}{$\begin{array}{c}2.251 \\
(0,134)\end{array}$} \\
\hline & & Porcentaje & $96,0 \%$ & $83,3 \%$ & $89,1 \%$ & \\
\hline & \multirow{2}{*}{ Mixta } & Frecuencia & 1 & 5 & 6 & \\
\hline & & Porcentaje & $4,0 \%$ & $16,7 \%$ & $10,9 \%$ & \\
\hline \multirow{2}{*}{\multicolumn{2}{|c|}{ Total }} & Frecuencia & 25 & 30 & 55 & \\
\hline & & Porcentaje & $100,0 \%$ & $100,0 \%$ & $100,0 \%$ & \\
\hline
\end{tabular}

La relación arrojó que el tipo de fatiga presente en los segmentos de hombro y muñeca, fue la fatiga física con un porcentaje de $89,5 \%$ de los 19 manifestados en hombre, y 83,3\% en muñeca de los 30 presentados. No se halló asociación estadística entre estas.

Tabla 14. Relación limitación hombro versus tipo de fatiga inicio de la jornada laboral.

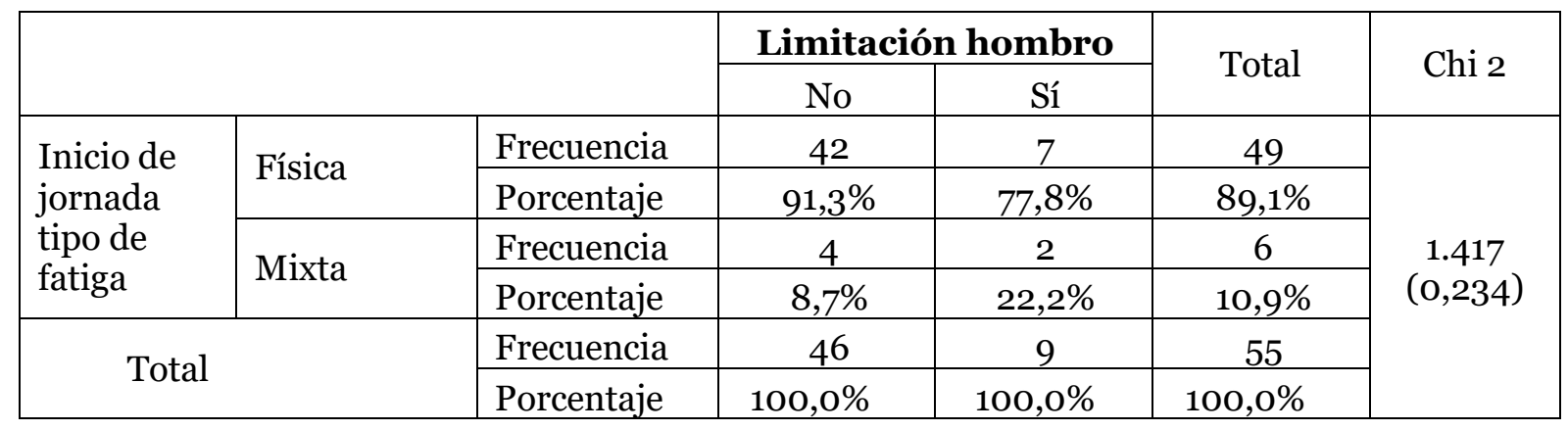


Tabla 15. Relación limitación muñeca versus tipo de fatiga inicio de la jornada laboral.

\begin{tabular}{|c|c|c|c|c|c|c|}
\hline & \multicolumn{2}{|c|}{ Limitación muñeca } & \multirow{2}{*}{ Total } & \multirow{2}{*}{ Chi 2} \\
\hline & & & No & Sí & & \\
\hline \multirow{4}{*}{$\begin{array}{l}\text { Inicio de } \\
\text { jornada } \\
\text { tipo de } \\
\text { fatiga }\end{array}$} & \multirow{2}{*}{ Física } & Frecuencia & 40 & 9 & 49 & \multirow{6}{*}{$\begin{array}{c}0.010 \\
(0,919)\end{array}$} \\
\hline & & Porcentaje & $88,9 \%$ & $90,0 \%$ & $89,1 \%$ & \\
\hline & \multirow{2}{*}{ Mixta } & Frecuencia & 5 & 1 & 6 & \\
\hline & & Porcentaje & $11,1 \%$ & $10,0 \%$ & $10,9 \%$ & \\
\hline \multirow{2}{*}{\multicolumn{2}{|c|}{ Total }} & Frecuencia & 45 & 10 & 55 & \\
\hline & & Porcentaje & $100,0 \%$ & $100,0 \%$ & $100,0 \%$ & \\
\hline
\end{tabular}

Se halló que la relación entre la limitación para las actividades en hombro y muñeca, están en una frecuencia entre 7 y 9, respecto del tipo de la fatiga física al inicio de la jornada laboral.

Tabla 16. Relación dolor hombro versus nivel de fatiga final de la jornada laboral.

\begin{tabular}{|c|c|c|c|c|c|c|}
\hline & \multicolumn{2}{|c|}{ Dolor hombro } & \multirow{2}{*}{ Total } & \multirow{2}{*}{ Chi 2} \\
\hline & & & No & Sí & & \\
\hline \multirow{6}{*}{$\begin{array}{l}\text { Nivel de } \\
\text { fatiga al } \\
\text { final } \\
\text { jornada }\end{array}$} & \multirow{2}{*}{ Leve } & Frecuencia & 1 & $\mathrm{O}$ & 1 & \multirow{8}{*}{$\begin{array}{c}0.968 \\
(0,616)\end{array}$} \\
\hline & & Porcentaje & $2,8 \%$ & $0,0 \%$ & $1,8 \%$ & \\
\hline & \multirow{2}{*}{ Moderada } & Frecuencia & 6 & 2 & 8 & \\
\hline & & Porcentaje & $16,7 \%$ & $10,5 \%$ & $14,5 \%$ & \\
\hline & \multirow{2}{*}{ Severa } & Frecuencia & 29 & 17 & 46 & \\
\hline & & Porcentaje & $80,6 \%$ & $89,5 \%$ & $83,6 \%$ & \\
\hline \multirow{2}{*}{\multicolumn{2}{|c|}{ Total }} & Frecuencia & 36 & 19 & 55 & \\
\hline & & Porcentaje & $100,0 \%$ & $100,0 \%$ & $100,0 \%$ & \\
\hline
\end{tabular}

Tabla 17. Relación dolor codo versus nivel de fatiga final de la jornada laboral.

\begin{tabular}{|c|c|c|c|c|c|c|}
\hline & & & \multicolumn{2}{|c|}{ Dolor codo } & \multirow{2}{*}{ Total } & \multirow{2}{*}{ Chi 2} \\
\hline & & & No & Sí & & \\
\hline \multirow{6}{*}{$\begin{array}{l}\text { Nivel de } \\
\text { fatiga al } \\
\text { final } \\
\text { jornada }\end{array}$} & \multirow{2}{*}{ Leve } & Frecuencia & 1 & 0 & 1 & \multirow{8}{*}{$\begin{array}{c}0.143 \\
(0,931)\end{array}$} \\
\hline & & Porcentaje & $2,0 \%$ & $0,0 \%$ & $1,8 \%$ & \\
\hline & \multirow{2}{*}{ Moderada } & Frecuencia & 7 & 1 & 8 & \\
\hline & & Porcentaje & $14,3 \%$ & $16,7 \%$ & $14,5 \%$ & \\
\hline & \multirow{2}{*}{ Severa } & Frecuencia & 41 & 5 & 46 & \\
\hline & & Porcentaje & $83,7 \%$ & $83,3 \%$ & $83,6 \%$ & \\
\hline \multirow{2}{*}{\multicolumn{2}{|c|}{ Total }} & Frecuencia & 49 & 6 & 55 & \\
\hline & & Porcentaje & $100,0 \%$ & $100,0 \%$ & $100,0 \%$ & \\
\hline
\end{tabular}


Tabla 18. Relación dolor muñeca versus nivel de fatiga final de la jornada laboral.

\begin{tabular}{|c|c|c|c|c|c|c|}
\hline & \multicolumn{2}{|c|}{ Dolor muñeca } & \multirow{2}{*}{ Total } & \multirow{2}{*}{ Chi 2} \\
\hline & & & No & Sí & & \\
\hline \multirow{6}{*}{$\begin{array}{l}\text { Nivel de } \\
\text { fatiga al } \\
\text { final } \\
\text { jornada }\end{array}$} & Leve & Frecuencia & 1 & 0 & 1 & \multirow{8}{*}{$\begin{array}{c}2.654 \\
(0,265)\end{array}$} \\
\hline & Leve & Porcentaje & $4,0 \%$ & $0,0 \%$ & $1,8 \%$ & \\
\hline & \multirow{2}{*}{ Moderada } & Frecuencia & 2 & 6 & 8 & \\
\hline & & Porcentaje & $8,0 \%$ & $20,0 \%$ & $14,5 \%$ & \\
\hline & \multirow{2}{*}{ Severa } & Frecuencia & 22 & 24 & 46 & \\
\hline & & Porcentaje & $88,0 \%$ & $80,0 \%$ & $83,6 \%$ & \\
\hline \multirow{2}{*}{\multicolumn{2}{|c|}{ Total }} & Frecuencia & 25 & 30 & 55 & \\
\hline & & Porcentaje & $100,0 \%$ & $100,0 \%$ & $100,0 \%$ & \\
\hline
\end{tabular}

Los datos arrojaron que, el nivel de fatiga severa es el que predomina en el dolor de los tres segmentos. No obstante, no se encontró asociación estadística entre estas.

Tabla 19. Relación limitación actividades en hombro versus nivel de fatiga final de la jornada laboral.

\begin{tabular}{|c|c|c|c|c|c|c|}
\hline & \multicolumn{2}{|c|}{ Limitación hombro } & \multirow{2}{*}{ Total } & \multirow{2}{*}{ Chi 2} \\
\hline & & & No & Sí & & \\
\hline \multirow{6}{*}{$\begin{array}{l}\text { Nivel de } \\
\text { fatiga al } \\
\text { final } \\
\text { jornada }\end{array}$} & \multirow{2}{*}{ Leve } & Frecuencia & 1 & 0 & 1 & \multirow{8}{*}{$\begin{array}{c}0.318 \\
(0,853)\end{array}$} \\
\hline & & Porcentaje & $2,2 \%$ & $0,0 \%$ & $1,8 \%$ & \\
\hline & \multirow{2}{*}{ Moderada } & Frecuencia & 7 & 1 & 8 & \\
\hline & & Porcentaje & $15,2 \%$ & $11,1 \%$ & $14,5 \%$ & \\
\hline & \multirow{2}{*}{ Severa } & Frecuencia & 38 & 8 & 46 & \\
\hline & & Porcentaje & $82,6 \%$ & $88,9 \%$ & $83,6 \%$ & \\
\hline \multirow{2}{*}{\multicolumn{2}{|c|}{ Total }} & Frecuencia & 46 & 9 & 55 & \\
\hline & & Porcentaje & $100,0 \%$ & $100,0 \%$ & $100,0 \%$ & \\
\hline
\end{tabular}

Tabla 2o. Relación limitación actividades en muñeca versus nivel de fatiga final de la jornada laboral.

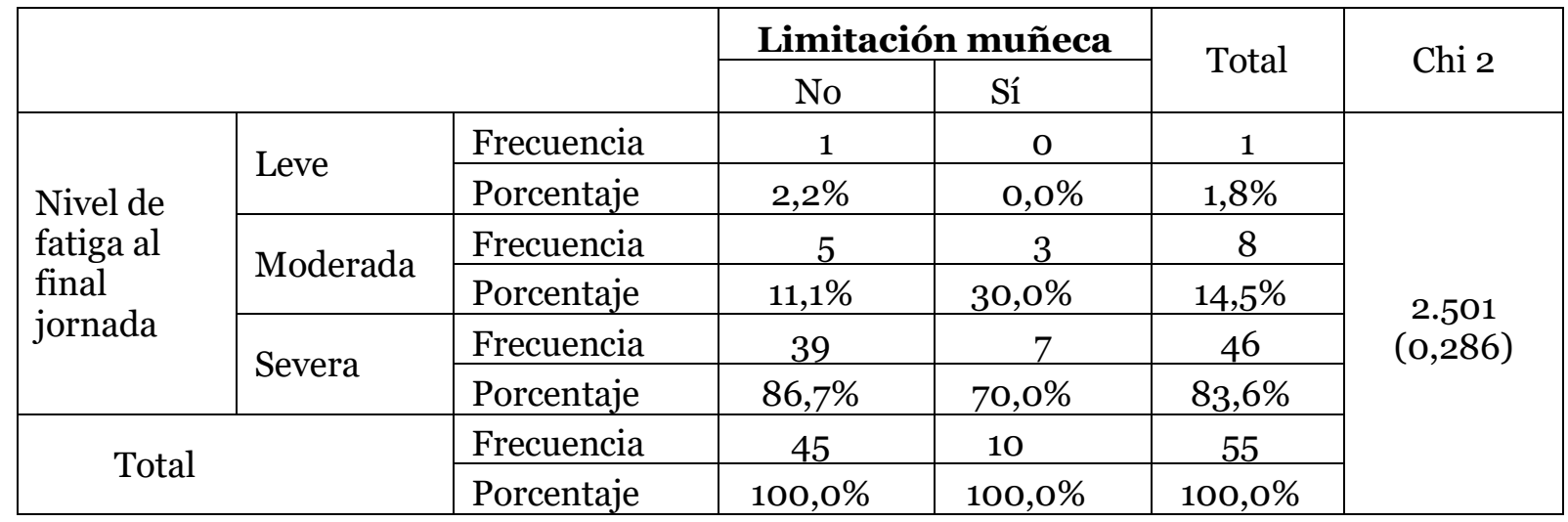

Se pudo evidenciar que la relación entre el nivel de fatiga al finalizar la jornada, es 
severo en aquellas operarias que manifestaron limitación en las actividades en los segmentos de hombro y muñeca

Tabla 21. Relación incapacidad en muñeca versus nivel de fatiga final de la jornada laboral.

\begin{tabular}{|c|c|c|c|c|c|c|}
\hline & \multicolumn{2}{|c|}{ Incapacidad muñeca } & \multirow{2}{*}{ Total } & \multirow{2}{*}{ Chi 2} \\
\hline & & & No & Sí & & \\
\hline \multirow{6}{*}{$\begin{array}{l}\text { Nivel de } \\
\text { fatiga al } \\
\text { final } \\
\text { jornada }\end{array}$} & \multirow{2}{*}{ Leve } & Frecuencia & 1 & 0 & 1 & \multirow{8}{*}{$\begin{array}{c}2.917 \\
(0,233)\end{array}$} \\
\hline & & Porcentaje & $2,0 \%$ & $0,0 \%$ & $1,8 \%$ & \\
\hline & \multirow{2}{*}{ Moderada } & Frecuencia & 6 & 2 & 8 & \\
\hline & & Porcentaje & $12,0 \%$ & $40,0 \%$ & $14,5 \%$ & \\
\hline & \multirow{2}{*}{ Severa } & Frecuencia & 43 & 3 & 46 & \\
\hline & & Porcentaje & $86,0 \%$ & $60,0 \%$ & $83,6 \%$ & \\
\hline \multirow{2}{*}{\multicolumn{2}{|c|}{ Total }} & Frecuencia & 50 & 5 & 55 & \\
\hline & & Porcentaje & $100,0 \%$ & $100,0 \%$ & $100,0 \%$ & \\
\hline
\end{tabular}

Se encontró que las operarias que presentaron incapacidad, debido al segmento de muñeca, tienen relación con fatiga moderada y severa al final de la jornada.

En relación al análisis comparativo entre las variables sintomatología dolorosa osteomuscular y fatiga laboral, como se observa en la mayoría de las tablas, existe una correlación entre fatiga severa, fatiga física y dolor, esto a pesar de no encontrarse significancia asociativa, lo que probablemente esté relacionado con el tamaño de la muestra.

\section{Propuestas basadas en criterios ergonómicos}

Esta propuesta se presenta, con base a los datos arrojados por los objetivos anteriores y a la recopilación de información en un estudio previo realizado en la empresa.

Tabla 22. Propuesta ergonómica factor exigencia de producción.

\begin{tabular}{|c|c|c|c|}
\hline $\begin{array}{l}\text { Factor de la } \\
\text { organización }\end{array}$ & Problema & Posible solución & Imagen \\
\hline $\begin{array}{l}\text { Exigencia de } \\
\text { producción }\end{array}$ & $\begin{array}{lr}\text { Se debe cumplir con } \\
\text { la entrega de ciertos } \\
\text { lotes diarios, lo que } \\
\text { supone de horas } \\
\text { laborales } & \text { extras. } \\
\text { Acorde con } & \text { la } \\
\text { temporada, } & \text { sin } \\
\text { embargo, para } & \text { la } \\
\text { mayoría de } & \text { las } \\
\text { operarias es una } \\
\text { oportunidad } \\
\text { mejorar sus ingresos. }\end{array}$ & $\begin{array}{l}\text { Optimizar la presión } \\
\text { de la producción, de } \\
\text { manera que haya un } \\
\text { ajuste entre las } \\
\text { demandas del } \\
\text { entorno, como lo es la } \\
\text { organización de los } \\
\text { arrumes de tela en el } \\
\text { espacio. Por otra } \\
\text { parte, implementar } \\
\text { recompensas, podría } \\
\text { resultar en un aspecto } \\
\text { motivacional. }\end{array}$ & $\begin{array}{llr} & & \\
\text { Arrume de prendas a } \\
\text { un lado y otro por } \\
\text { aumento } & \text { de } & \text { la } \\
\text { producción. } & & \end{array}$ \\
\hline
\end{tabular}


Tabla 23. Propuesta ergonómica factor innovación tecnológica.

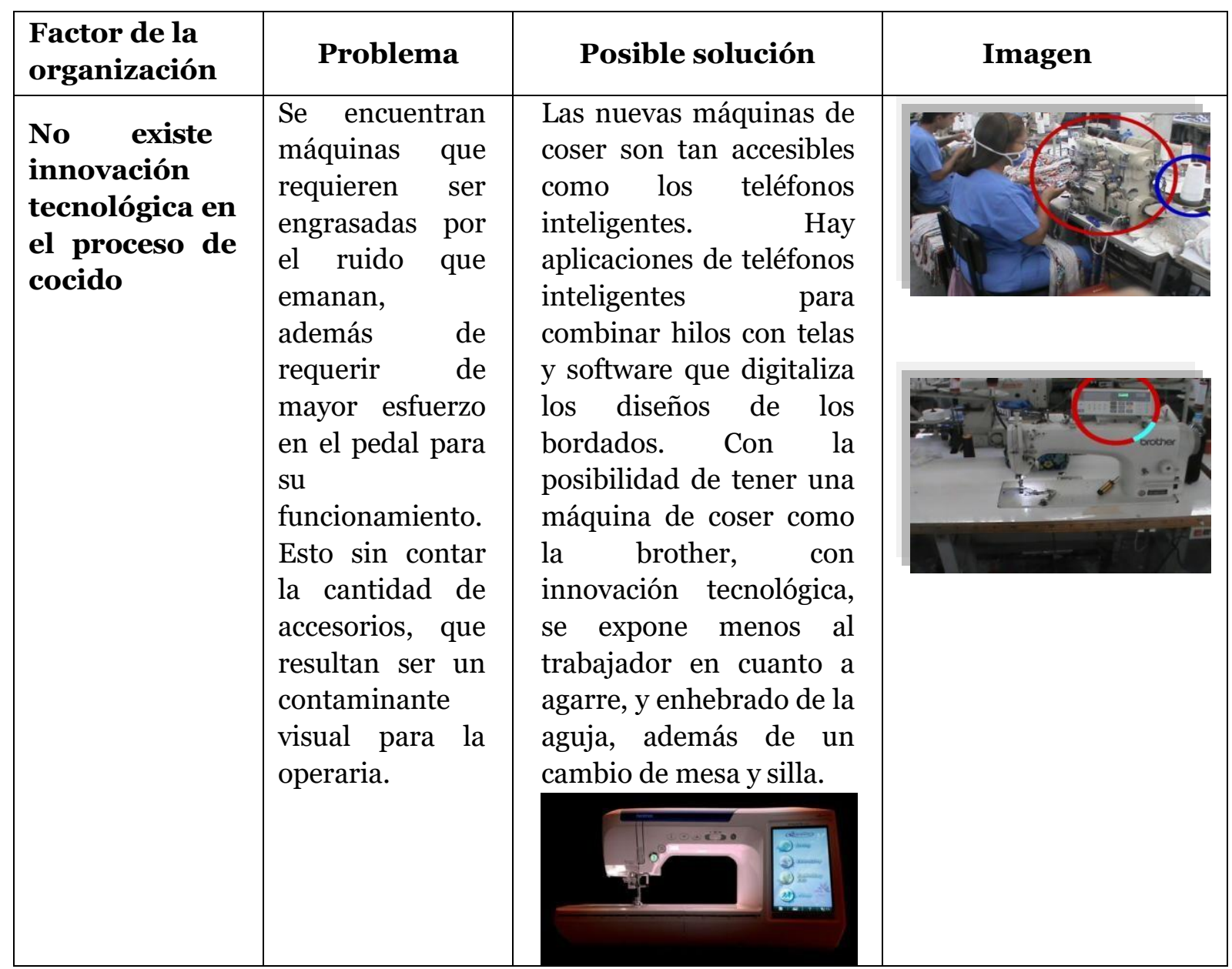

Tabla 24. Propuesta ergonómica factores físicos y locativos.

\begin{tabular}{|c|c|c|c|}
\hline $\begin{array}{l}\text { Factor de la } \\
\text { organización }\end{array}$ & Problema & Posible solución & Imagen \\
\hline $\begin{array}{l}\text { Físicosy } \\
\text { locativos } \\
\text { iluminación } \\
\text { espacio }\end{array}$ & $\begin{array}{l}\text { Se presenta } \\
\text { fatiga visual, } \\
\text { probablemente } \\
\text { relacionada con } \\
\text { la iluminación } \\
\text { deficiente } \\
\text { general y la } \\
\text { luminaria } \\
\text { adaptada que } \\
\text { tienen algunas } \\
\text { máquinas, las } \\
\text { cuales no } \\
\text { permiten una } \\
\text { luminiscencia } \\
\text { adecuada al } \\
\text { momento de }\end{array}$ & $\begin{array}{l}\text { Las fuentes de luz deben } \\
\text { colocarse de manera que } \\
\text { eviten los } \\
\text { deslumbramientos y los } \\
\text { reflejos molestos en la } \\
\text { pantalla de las máquinas } \\
\text { y la base de estas. } \\
\text { Se deben garantizar los } \\
\text { niveles de iluminación } \\
\text { recomendados, en } \\
\text { función del tipo de tarea, } \\
\text { y si fuese necesario, } \\
\text { instalar iluminación }\end{array}$ & \\
\hline
\end{tabular}




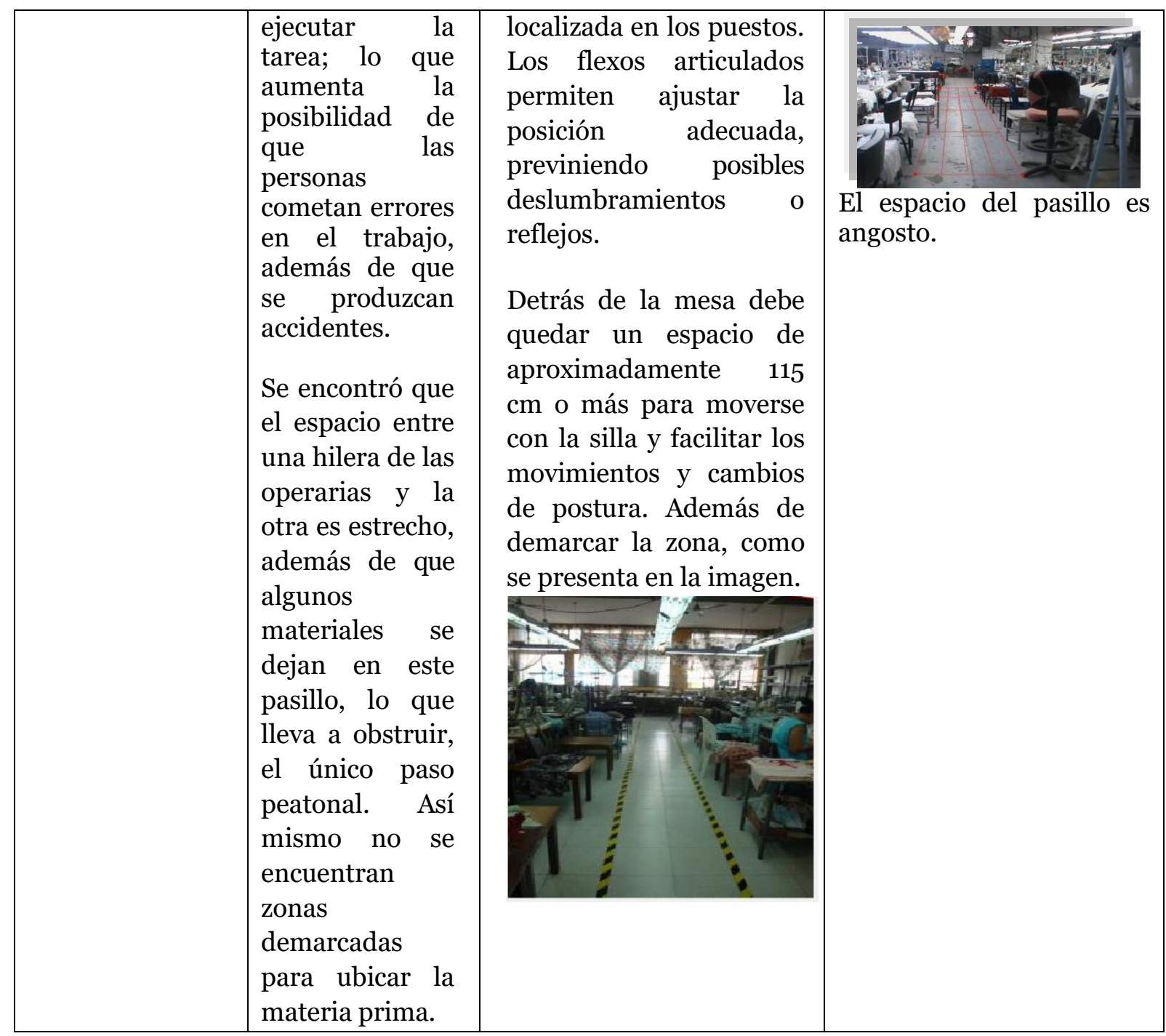

Tabla 25. Propuesta ergonómica factores ambientales.

\begin{tabular}{|l|l|l|l|}
\hline $\begin{array}{l}\text { Factor de la } \\
\text { organización }\end{array}$ & \multicolumn{1}{|c|}{ Problema } & Posible solución & \multicolumn{1}{|c|}{ Imagen } \\
\hline $\begin{array}{l}\text { Ambientales } \\
\text { (ruido, } \\
\text { temperatura) }\end{array}$ & $\begin{array}{l}\text { Percepción de } \\
\text { disconfort térmico } \\
\text { y acústico, } \\
\text { principalmente en } \\
\text { el horario de la } \\
\text { tarde, debido al } \\
\text { material del techo } \\
\text { (eternit), el calor } \\
\text { emanado por las } \\
\text { máquinas, y al antenimiento del } \\
\text { número dañado, } \\
\text { personas. }\end{array}$ & $\begin{array}{l}\text { lector de otro y } \\
\text { controlar los EPP, } \\
\text { además de aceitar las } \\
\text { máquinas de coser. }\end{array}$ & $\begin{array}{l}\text { El extractor se encuentra } \\
\text { dañado, además de ser } \\
\text { insuficiente en el área. }\end{array}$ \\
\hline
\end{tabular}


Tabla 26. Propuesta ergonómica factores biomecánicos.

\begin{tabular}{|c|c|c|c|}
\hline $\begin{array}{l}\text { Factor de la } \\
\text { organización }\end{array}$ & Problema & Posible solución & Imagen \\
\hline $\begin{array}{l}\text { Biomecánicos: } \\
\text { Postura } \\
\text { mantenida, } \\
\text { movimiento } \\
\text { repetitivo }\end{array}$ & 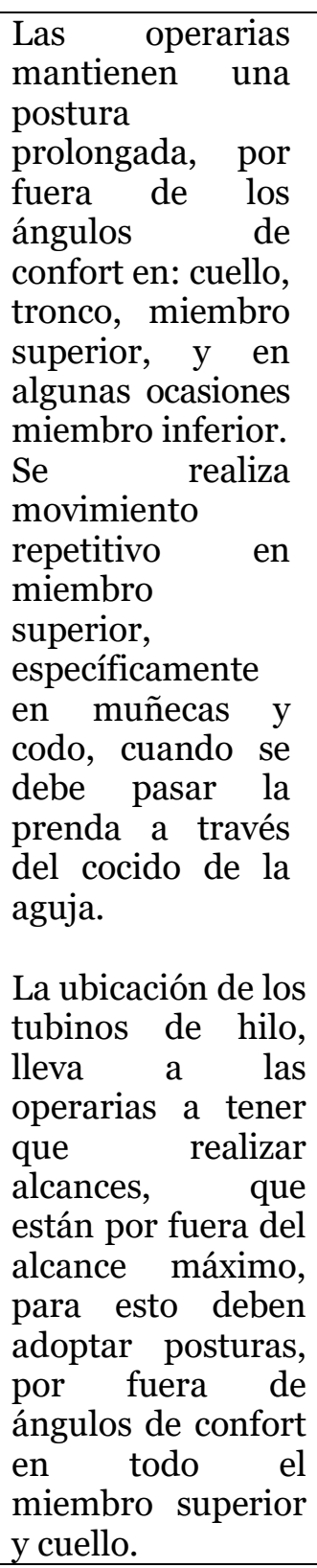 & 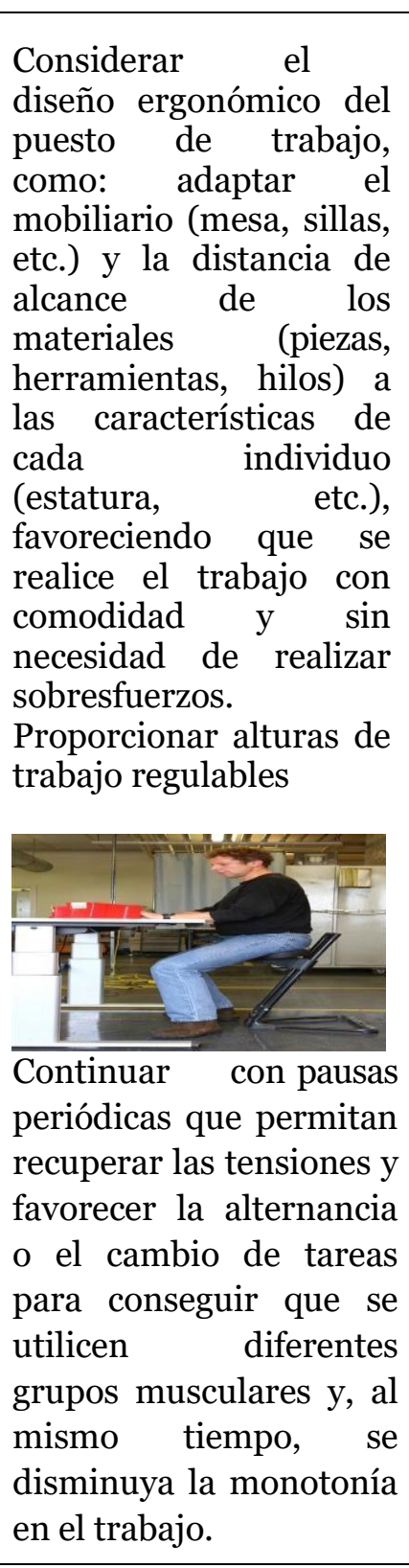 & $\begin{array}{l}\text { Postura mantenida de } \\
\text { antebrazo sostenido sobre } \\
\text { el borde cortante de la } \\
\text { mesa. }\end{array}$ \\
\hline
\end{tabular}

\section{Conclusiones}

La multiplicidad de los factores que influyen en la aparición de sintomatología dolorosa en miembro superior, hace que no solo la intensidad de la tarea, la frecuencia, los movimientos repetitivos y las posturas mantenidas se asocien con su presencia, sino que otros factores como los organizacionales estén jugando un papel determinante en la génesis de los desórdenes osteomusculares. 
Los resultados del presente estudio muestran que la población, estuvo conformada por mujeres en edad productiva, quienes desempeñan labores de manufactura textil, ocupación en la cual realizan movimientos repetitivos de las muñecas y de los dedos, acompañados de contracción estática en los músculos de cuello y espalda, que se transforman con el pasar de los años en tensión muscular, incrementando el riesgo de presentar desordenes musculoesqueléticos.

La aplicación de los instrumentos permitió identificar la prevalencia de dolor y de fatiga laboral manifestado por las operarias, y adicionalmente permitió caracterizar la sintomatología osteomuscular en términos de segmento, incapacidad, de tipo y nivel de fatiga laboral, lo cual puede ser usado como información epidemiológica para realizar planes de intervención.

Se encontró que la única asociación significativa arrojada por las tablas de contingencia estaba dada por las variables incapacidad en hombro versus nivel de fatiga al inicio de la jornada.

La sintomatología dolorosa osteomuscular y la fatiga laboral es frecuente en esta población, siendo el segmento de hombro y muñeca los más comprometidos, además de que el nivel y tipo de fatiga predominante, fueron la severa y la física, esto probablemente como resultado de los esfuerzos, posturas sostenidas y movimientos repetidos, además de la jornada laboral presente en la población estudiada.

Existen por parte de la empresa, varios factores ergonómicos a considerar desde lo organizacional, como son: factores individuales, físicos, ambientales, locativos, herramientas, máquinas, posturas, movimientos, cognitivos y políticas de la empresa, entre otros.

\section{Referencias}

Aguayo-Canela, M. (2007). Cómo realizar "paso a paso" un contraste de hipótesis con SPSS para Windows: (III) Relación o asociación y análisis de la dependencia (o no) entre dos variables cuantitativas. Correlación y regresión lineal simple. https://statistics.blogs.uv.es/files/2014/o6/Correlaci\%C3\%B3n-y-regresi\%C3\%B3n.pdf

Araujo, V., \& Alhejandra, M. (2014). Fatiga laboral en trabajadores de una ensambladora de vehículos. Valencia, 2012-2013 [tesis de postgrado]. Repositorio Institucional de la Universidad de Carabobo. http://mriuc.bc.uc.edu.ve/bitstream/handle/123456789/500/maraujo.pdf?sequence $=4$

Bichachi, D. S. (2004). El uso de las listas de chequeo (CHEK-LIST) como herramienta para controlar la calidad de las leyes. https://www.claudiabernazza.com.ar/htm/pdf/check list.pdf

Cubillos, C.M.N., \& López, Y.A.R. (2009). Fatiga laboral, accidentes e incidentes laborales en los conductores de carga pesada de una empresa transportista de la ciudad de Yopal. Cuadernos Hispanoamericanos de Psicología, 10(1), 7-21. https://silo.tips/download/fatiga-laboralaccidentes-e-incidentes-laborales-en-los-conductores-de-carga-pes 
Escorza, J.V. (2010). Bichachi, [tesis de maestría]. Repositorio Dspace. https://tesis.ipn.mx/handle/123456789/7517

Gómez-Cajas, L. D. (2014). Estudio de la fatiga laboral en los funcionarios de la procuraduría general del estado (DR1) [tesis de pregrado]. Repositorio Institucional de la Universidad de Guayaquil. http://repositorio.ug.edu.ec/handle/redug/13648

Rengifo-Villavicencio, A. (2020). Síntomas musculoesqueléticos en el personal asistencial del CAP III Iquitos Essalud, utilizando el cuestionario nordico de kuorinka estandarizado [tesis de pregrado]. Repositorio Institucional de la Universidad Científica del Perú. http://repositorio.ucp.edu.pe/handle/UCP/977 